\title{
Leading Yukawa corrections to Higgs production associated with a tagged bottom-antibottom pair in the standard model at the CERN LHC
}

\author{
F. Boudjema ${ }^{1}$ and Le Duc Ninh ${ }^{1,2}$ \\ ${ }^{1}$ LAPTH, Université de Savoie, CNRS, BP 110, F-74941 Annecy-le-Vieux Cedex, France \\ ${ }^{2}$ Theory Division, CERN, CH-1211 Geneva 23, Switzerland \\ (Received 21 November 2007; published 15 February 2008)
}

\begin{abstract}
Considering the large value of the top Yukawa coupling, we investigate the leading one-loop Yukawa electroweak corrections that can be induced by the top quark in a process such as Higgs production in association with a tagged bottom-antibottom pair at the LHC. At next-to-leading order these contributions are found to be small at the LHC both for the total cross section and for the distributions. In the limit of vanishing bottom Yukawa coupling where the leading order contribution vanishes, the process can still be induced at one-loop through the top quark transition. Though this contribution which can be counted as part of the next-to-next-to-leading order correction is small for Higgs masses around $120 \mathrm{GeV}$, it quickly picks up for higher Higgs masses. This contribution represents the rescattering of the top quarks and their decay into $W$ 's leading to Higgs production through $W W$ fusion.
\end{abstract}

DOI: 10.1103/PhysRevD.77.033003

PACS numbers: $14.80 . \mathrm{Bn}, 12.15 . \mathrm{Lk}$

\section{INTRODUCTION}

The most important goal of the Large Hadron Collider (LHC) is the discovery of the Higgs and the concomitant study of the mechanism of electroweak (EW) symmetry breaking. Especially if no new phenomenon is unraveled through the direct production of new particles, the study of the Higgs properties such as its self-couplings and couplings to the other particles of the standard model (SM) will be crucial in order to establish the nature of the scalar component of the model. In this respect most prominent couplings, in the SM, are the Higgs, the top, and to a much lesser degree the bottom, Yukawa couplings. The top Yukawa coupling is after all of the order of the strong QCD coupling and plays a crucial role in a variety of Higgs related issues. The dominant mechanism for Higgs production at the Large Hadron Collider is the gluon fusion process, which incidentally is initiated through a top loop. Electroweak gauge boson fusion and $W / Z H$ associated production [1] are also of importance. Higgs production associated with heavy quarks like the top or bottom quark is not considered as a discovery channel because of its small total cross section, the top suffering further from a complicated final state topology. However, if one wants to determine the bottom-Higgs Yukawa coupling, $\lambda_{b b H}$, then Higgs production associated with a bottom-antibottom pair could provide a direct measurement of this coupling. In the minimal supersymmetric standard model (MSSM) the bottom Yukawa coupling is enhanced by a factor $\tan \beta$, the ratio of the vacuum expectation values of the two Higgs doublets. For high $\tan \beta$ and not too large Higgs masses this provides an important discovery channel for the supersymmetric Higgses.

The next-to-leading order (NLO) QCD correction to $p p \rightarrow b \bar{b} H$ has been calculated by different groups relying on different formalisms. In a nutshell, in the five-flavor scheme (5FNS) [2,3], use is made of the bottom distribu- tion function so that the process is approximated [at leading order (LO)] by the fusion $b \bar{b} \rightarrow H$. This gives an approximation to the inclusive cross section dominated by the untagged low $p_{T}$ outgoing $b$ jets. If only one final $b$ is tagged, the cross section is approximated by $g b \rightarrow b H$. The four flavor scheme (4FNS) has no $b$ parton initiated process but is induced by gluon fusion $g g \rightarrow b \bar{b} H$, with a very small contribution from the light quark initiated process $q \bar{q} \rightarrow b \bar{b} H .{ }^{1}$ Here again the largest contribution is due to low $p_{T}$ outgoing $b$ 's which can be accounted for by gluon splitting into $b \bar{b}$. The latter needs to be resummed and hence one recovers most of the 5FNS calculation while retaining the full kinematics of the reaction. QCD NLO corrections have been performed in both schemes [3-6] and one has now reached a quite good agreement [7].

The 5FNS approach, which at leading order is a two-toone process has allowed the computation of the next-tonext-to-leading order (NNLO) QCD correction $[8,9]$ and very recently the electroweak/supersymmetry (SUSY) correction [10] to $b \bar{b} \rightarrow \phi, \phi$ any of the neutral Higgs boson in the MSSM. SUSY QCD corrections have also been performed for $g g \rightarrow b \bar{b} h[11,12]$ where $h$ is the lightest Higgs in the MSSM as well as to $g b \rightarrow b \phi$ [13].

In order to exploit this production mechanism to study the Higgs couplings to $b$ 's, one must identify the process and therefore one needs to tag both $b$ 's, requiring somewhat large $p_{T} b$. This reduces the cross section but gives much better signal over background ratio. For large $p_{T}$ outgoing quarks one needs to rely on the 4FNS to properly reproduce the high $p_{T} b$ quarks. The aim of this paper is to report on the calculation of the leading electroweak cor-

\footnotetext{
${ }^{1}$ In fact $q \bar{q} \rightarrow b \bar{b} H$ is dominated by $q \bar{q} \rightarrow H Z^{*} \rightarrow b \bar{b} H$ and does not vanish for vanishing bottom Yukawa coupling. However this contribution should be counted as $\mathrm{ZH}$ production and can be excluded by imposing an appropriate cut on the invariant mass of the $b \vec{b}$ pair.
} 
rections to the exclusive $b b H$ final state, meaning two $b$ 's are detected. These leading electroweak corrections are triggered by top-charged Goldstone loops whereby, in effect, an external $b$ quark turns into a top. This transition has a specific chiral structure whose dominant part is given by the top mass or, in terms of couplings, to the top Yukawa coupling. Considering that the latter is of the order of the QCD coupling constant, the corrections might be large. In fact, as we shall see, such type of transitions can trigger $g g \rightarrow b \bar{b} H$ even with vanishing $\lambda_{b b H}$ in which case the process is generated solely at one-loop. We will quantify the effect of such contributions.

This calculation belonging to the class of the $2 \rightarrow 3$ processes at the LHC, we will also cover some technical issues pertaining to such calculations, like among other things the helicity amplitude method we use and the occurrence of vanishingly small (inverse) Gram determinant. This determinant occurs when solving the system of (linearly independent) tensor integrals in terms of the basis of scalar integrals. The Gram matrix is constructed out of the scalar products of the $(N-1)$ linearly independent momenta for a process with $N$ external legs. The Gram determinant can vanish if the momenta of the set are, for example, for some exceptional point in phase space no longer linearly independent.

In this paper we restrict ourselves to a Higgs mass in the range preferred by the latest electroweak data [14]; in particular, we confine the present study to $M_{H}<150 \mathrm{GeV}$. Another reason for this choice is that, as we will briefly point out, as the Higgs mass increases the loop induced cross section increases and the loop integral starts showing instabilities. This we have identified as a Landau singularity which is a pinch singularity of the loop integral. This has an interesting physical origin: the rescattering of onshell top quarks into $W$ bosons, giving rise to $W$ boson fusion into Higgs. We leave this important issue to another study though.

The plan of the paper is as follows. In the next section we present some general considerations concerning the properties and structure of the calculation we have performed. We first briefly review the tree-level LO amplitude and highlight some symmetries of the helicity amplitudes. These symmetries are maintained by QCD corrections but not by the electroweak corrections we are studying. We then discuss the leading approximation as given by the insertions of the top-bottom-Goldstone Yukawa vertex. We classify the contributions into three classes in the cases of the NLO correction as well as the contributions that survive at one-loop even for $\lambda_{b b H}=0$. In Sec. III we give our renormalization scheme and discuss the inclusion of a top/ Higgs Yukawa enhanced contribution which can be considered as a universal correction to Higgs processes related to the Higgs wave function renormalization and the renormalization of the vacuum expectation value. Section IV gives an overview of some calculational details, in particular, how the calculation is organized. Discussion on the loop integrals, the appearance of spurious instabilities related to vanishing Gram determinants, and how these are cured depending on how the phase space integration is carried out is also presented. We also discuss in this section how we checked our results through ultraviolet finiteness and gauge invariance. Section V presents and discusses the numerical results we find for the total cross section and various distributions both at the level of the NLO electroweak correction as well as the one-loop contribution that survives in the limit of vanishing $\lambda_{b b H}$. Details about the helicity amplitude method we used as well as the optimization of the code are presented in the two appendices.

\section{GENERAL CONSIDERATIONS}

Before discussing the details of the calculation it is educative to expose some key features that appear when one considers the electroweak corrections at one-loop compared to the structure we have at tree-level or even the structure that emerges from QCD loop calculations. In particular the helicity structure is quite telling. So let us set our definition first. The process we consider is $g\left(p_{1}, \lambda_{1}\right)+$ $g\left(p_{2}, \lambda_{2}\right) \rightarrow b\left(p_{3}, \lambda_{3}\right)+\bar{b}\left(p_{4}, \lambda_{4}\right)+H\left(p_{5}\right) . \lambda_{i}= \pm$ with $i=1,2,3,4$ are the helicities of the gluons, the bottom, and antibottom while $p_{i}$ are the momenta of particles. The corresponding helicity amplitude will be denoted by $\mathcal{A}\left(\lambda_{1}, \lambda_{2} ; \lambda_{3}, \lambda_{4}\right)$.

\section{A. Leading order considerations}

At tree-level, see Fig. 1 for the contributing diagrams, the Higgs can only attach to the $b$ quark and therefore each diagram, and hence the total amplitude, is proportional to the Higgs coupling to $b \bar{b}, \lambda_{b b H}$. Compared to the gluon coupling this scalar coupling breaks chirality. These features remain unchanged when we consider QCD correc-
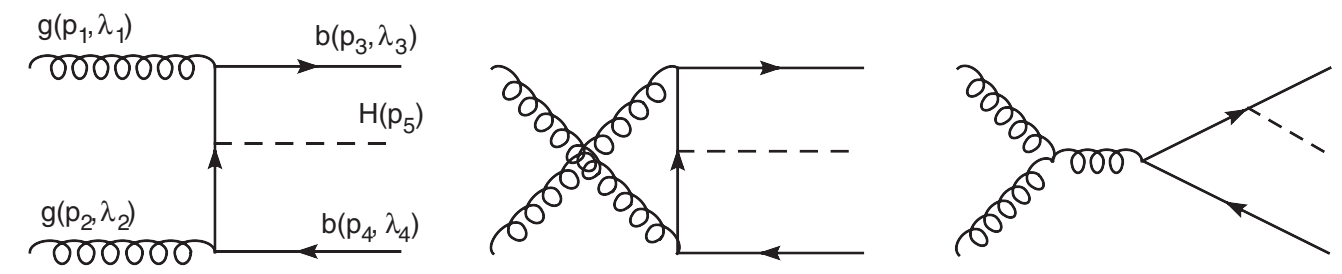

FIG. 1. All the eight Feynman diagrams can be obtained by inserting the Higgs line to all possible positions in the bottom line. 
tions. Moreover the QCD coupling and the Higgs coupling are parity conserving which allows to relate the state with helicities $\left(\lambda_{1}, \lambda_{2} ; \lambda_{3}, \lambda_{4}\right)$ to the one with $\left(-\lambda_{1},-\lambda_{2}\right.$; $\left.-\lambda_{3},-\lambda_{4}\right)$ therefore cutting by half the number of helicity amplitudes to calculate. With our conventions for the definition of the helicity states, see Appendix A, parity conservation for the tree-level helicity amplitude gives

$$
\mathcal{A}_{0}\left(-\lambda_{1},-\lambda_{2} ;-\lambda_{3},-\lambda_{4}\right)=\lambda_{3} \lambda_{4} \mathcal{A}_{0}\left(\lambda_{1}, \lambda_{2} ; \lambda_{3}, \lambda_{4}\right)^{\star} \text {. }
$$

This can be generalized at higher order in QCD with due care of possible absorptive parts in taking complex conjugation.

The number of contributing helicity amplitudes can be reduced even further at the leading order, in fact halved again, in the limit where one neglects the mass of the $b$ quark that originates from the $b$-quark spinors and therefore from the $b$ quark propagators. We should in this case consider the $\lambda_{b b H}$ as an independent coupling, intimately related to the model of symmetry breaking. In this case chirality and helicity arguments are the same, the $b$ and $\bar{b}$ must have opposite helicities for the leading order amplitudes, and hence only $\mathcal{A}_{0}\left(\lambda_{1}, \lambda_{2} ; \lambda,-\lambda\right)$ remain nonzero. In this limit, this means that only a string containing an even number of Dirac $\gamma$ matrices, which we will label in general as $\Gamma^{\text {even }}$ as opposed to $\Gamma^{\text {odd }}$ for a string with an odd number of $\gamma$ 's, can contribute.

In the general case and reinstating the $b$ mass, we may write the helicity amplitudes as

$$
\begin{aligned}
\mathcal{A}\left(\lambda_{1}, \lambda_{2} ; \lambda_{3}, \lambda_{4}\right)= & \bar{u}\left(\lambda_{3}\right)\left(\Gamma_{\lambda_{1}, \lambda_{2}}^{\text {even }}+\Gamma_{\lambda_{1}, \lambda_{2}}^{\text {odd }}\right) v\left(\lambda_{4}\right) \\
= & \delta_{\lambda_{3},-\lambda_{4}}\left(\mathcal{A}^{\text {even }}+m_{b} \tilde{\mathcal{A}}^{\text {odd }}\right) \\
& +\delta_{\lambda_{3}, \lambda_{4}}\left(\mathcal{A}^{\text {odd }}+m_{b} \tilde{\mathcal{A}}^{\text {even }}\right) .
\end{aligned}
$$

The label "even" in $\mathcal{A}^{\text {even }}$ and $\tilde{\mathcal{A}}^{\text {even }}$ are the contributions of $\Gamma^{\mathrm{even}}$ to the amplitude and likewise for "odd." This way of writing shows that $m_{b}$ originates from the mass insertion coming from the massive spinors and is responsible for chirality flip. In the limit $m_{b} \rightarrow 0, \Gamma_{\lambda_{1}, \lambda_{2}}^{\text {even }}$ and $\Gamma_{\lambda_{1}, \lambda_{2}}^{\text {odd }}$ contribute to different independent helicity amplitudes. In general $\Gamma^{\text {even }}$ and $\Gamma^{\text {odd }}$ differ by a (fermion) mass insertion. In fact $\Gamma^{\text {odd }}$ is proportional to a fermion mass insertion from a propagator. At leading order the mass insertion is naturally $m_{b}$, such that $\Gamma^{\text {odd }}$ is $\mathcal{O}\left(m_{b}\right)$. This shows that at leading order, corrections from $m_{b}=0$ to the total cross section are of order $\mathcal{O}\left(m_{b}^{2}\right)$. Of course there might be some enhancement of the $\mathcal{O}\left(m_{b}^{2}\right)$ terms if one remembers that the cross section can bring about terms of order $m_{b}^{2} /\left(p_{T}^{b}\right)^{2}$. However, in our calculation where we require the $b$ 's to be observed hence requiring a $p_{T}^{b}$ cut, the effect will be minimal. With $m_{b}=4.62 \mathrm{GeV}$, the effect of neglecting $m_{b}$ is that the cross section is increased by $3.7 \%$ for $\left|\mathbf{p}_{T}^{b, \bar{b}}\right|>20 \mathrm{GeV}$ and $1.1 \%$ for $\left|\mathbf{p}_{T}^{b, \bar{b}}\right|>50 \mathrm{GeV}$. At oneloop, the chiral structure of the weak interaction and the contribution of the top change many of the characteristics that we have just discussed for the tree-level.

\section{B. New electroweak Yukawa-type contributions, novel characteristics}

Indeed, look at the two contributions arising from the one-loop electroweak corrections given in Fig. 2. Now the Higgs can attach to the top or to the $W$. Therefore these contributions do not vanish in the limit $\lambda_{b b H}=0$. Because now the fermion loop is a top loop, the mass insertion in what we called $\Gamma^{\text {odd }}$ is proportional to the top mass and is not negligible. In fact the diagrams in Fig. 2 show the charged Goldstone boson in the loop. The latter triggers a $t \rightarrow b \chi_{W}$ transition whose dominant coupling is proportional to the Yukawa coupling of the top. We will in fact be working in the approximation of keeping only the Yukawa couplings. This reduces the number of diagrams and if working in the Feynman gauge as we do in this computation, only the Goldstone contributions survive. The neutral Goldstone bosons can only contribute corrections of order $\lambda_{b}^{2}$. We will neglect these $\mathcal{O}\left(\lambda_{b}^{2}\right)$ contributions at the amplitude level. However the order $\mathcal{O}\left(\lambda_{b}\right)$ corrections will be kept. All the corrections are then triggered by $t \rightarrow b \chi_{W}$, and apart from the QCD $g \rightarrow b \bar{b}$ vertex, only the Yukawa vertices shown in Fig. 3 below are needed to build up the full set of electroweak corrections.

Note that in models outside the standard model, the Higgs coupling to the fermion $f, \lambda_{f f H}$, can involve other parameters beside the corresponding Yukawa coupling $\lambda_{f}$. The Higgs coupling to the charged Goldstone involves the Higgs self-coupling or Yukawa coupling of the Higgs, $\lambda=$ $M_{H}^{2} / 2 v^{2}$ proportional to the square of the Higgs mass. The latter can be large for large Higgs masses. These considerations allow us to classify the contributions into three gauge invariant classes.

\section{Three classes of diagrams and the chiral structure at one-loop}

All the one-loop diagrams are classified into three gauge invariant groups as displayed in Fig. 4. The Higgs couples to the bottom quark in the first group [Fig. 4(a)], to the top quark in the second group [Fig. 4(b)], and to the charged Goldstone boson in the third group [Fig. 4(c)]. As shown in Fig. 4 each class can be efficiently reconstructed from the
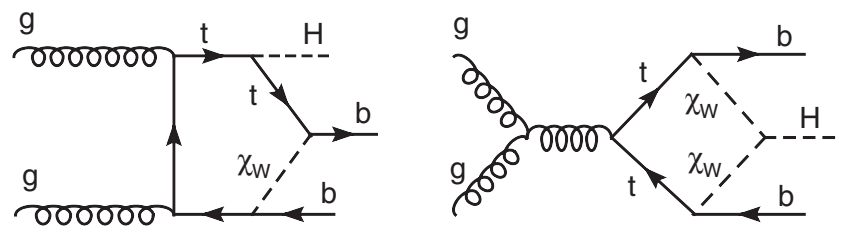

FIG. 2. Sample of one-loop diagrams related to the Yukawa interaction in the SM. $\chi_{W}$ represents the charged Goldstone boson. 

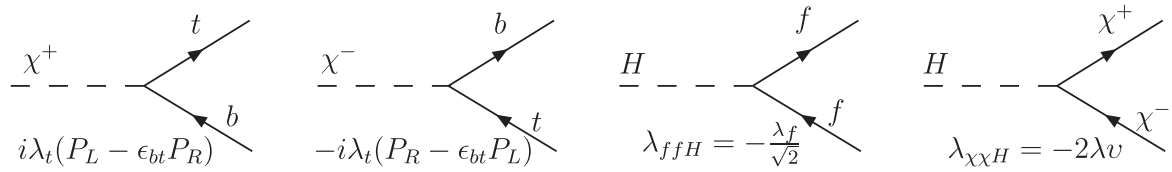

FIG. 3. Relevant vertices appearing at one-loop. $\varepsilon_{b t}=\lambda_{b} / \lambda_{t}, v$ is the vacuum expectation value, and $\lambda$ is the Higgs self-coupling, related to the Higgs mass in the standard model. $P_{L, R}=\left(1 \mp \gamma_{5}\right) / 2$.

(a)

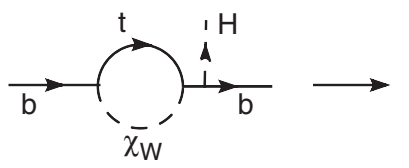

(b)

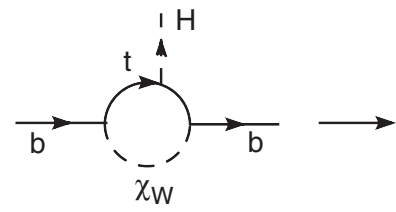

(c)

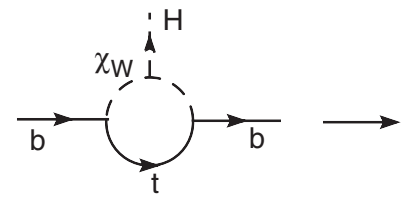

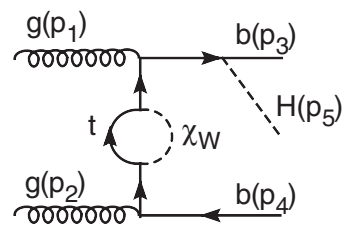
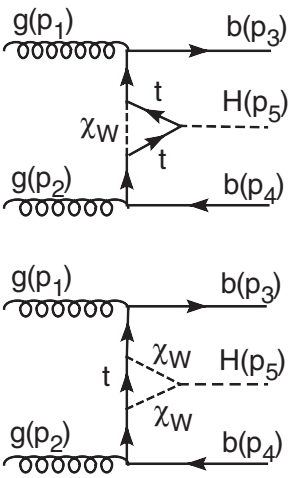
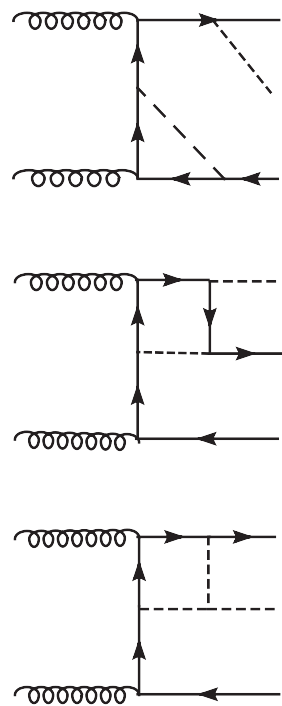
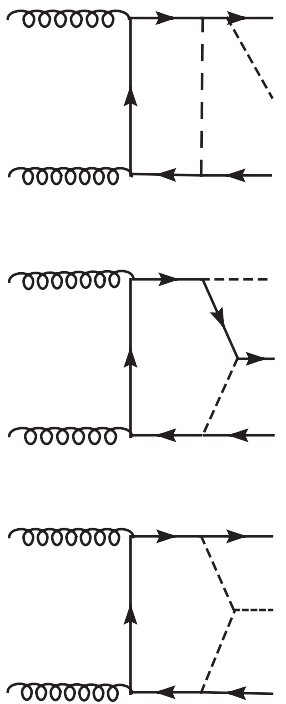

FIG. 4. All the diagrams in each group can be obtained by inserting the two gluon lines or one triple gluon vertex (not shown) to all possible positions in the generic bottom line, which is the first diagram on the left. We have checked the number of diagrams through GRACE-LOOP [15].

one-loop vertex $b \bar{b} H$, depending on which leg one attaches the Higgs, by then grafting the gluons in all possible ways. We have also checked explicitly that each class with its counterterms, see below, constitutes a QCD gauge invariant subset. Note that these three contributions depend on different combinations of independent couplings and therefore constitute independent sets.

The chiral structure $t \rightarrow b \chi_{W}$ impacts directly on the structure of the helicity amplitudes at one-loop. The split of each contribution according to $\Gamma^{\mathrm{even}}$ and $\Gamma^{\text {odd }}$, see Eq. (2), will turn out to be useful and will indicate which helicity amplitude can be enhanced by which Yukawa coupling at one-loop. We show only one example in class (b) of Fig. 4. It is straightforward to carry the same analysis for all other diagrams. We choose the first diagram in group (b) in Fig. 4. For clarity we will here take $m_{b}=0$, we have already shown how $m_{b}$ insertions are taken into account; see Eq. (2). Leaving aside the color part which can always be factorized out (see Appendix B) and the strong coupling constant, we write explicitly the contribution of this diagram as

$$
\begin{aligned}
\mathcal{A}_{b 1}\left(\lambda_{1}, \lambda_{2} ; \lambda_{3}, \lambda_{4}\right)= & \lambda_{t t H} \lambda_{t}^{2} \bar{u}\left(\lambda_{3}, p_{3}\right) \notin\left(\lambda_{1}, p_{1}\right) \\
& \times \frac{\bar{p}_{13}}{\bar{p}_{13}^{2}} C_{b 1} \frac{\bar{\not}_{24}}{\bar{p}_{24}^{2}} \notin\left(\lambda_{2}, p_{2}\right) u\left(\lambda_{4}, p_{4}\right) .
\end{aligned}
$$

$C_{b 1}$ is the Yukawa vertex correction. In $D$ dimension, with $q$ the integration variable, the momenta as defined in Fig. 1 with $p_{i j}=p_{i}+p_{j}$ and $\bar{p}_{i j}=p_{j}-p_{i}$, we have

$$
C_{b 1}=\int \frac{d^{D} q}{(2 \pi)^{D} i} \frac{\left(P_{R}-\varepsilon_{b t} P_{L}\right)\left(m_{t}+\not q+\bar{p}_{13}\right)\left(m_{t}+\not q-\bar{p}_{24}\right)\left(P_{L}-\varepsilon_{b t} P_{R}\right)}{\left(M_{W}^{2}-q^{2}\right)\left[m_{t}^{2}-\left(q+\bar{p}_{13}\right)^{2}\right]\left[m_{t}^{2}-\left(q-\bar{p}_{24}\right)^{2}\right]} .
$$

The numerator of the integrand of (4), neglecting terms of $\mathcal{O}\left(\lambda_{b}^{2}\right)$, can be rearranged such as 


$$
\begin{aligned}
\mathcal{A}_{b 1}\left(\lambda_{1}, \lambda_{2} ; \lambda_{3}, \lambda_{4}\right) \stackrel{\text { numerator }}{\longrightarrow} & \underbrace{-\varepsilon_{\text {even }}}_{\Gamma_{b t}\left(m_{t}^{2}+\left(\not q+\bar{p}_{13}\right)\left(\not q-\bar{\not}_{24}\right)\right)} \\
& +\underbrace{m_{t} P_{R}\left(2 \not 1+\bar{\not}_{13}-\overline{\not p}_{24}\right)}_{\Gamma^{\text {odd }}}
\end{aligned}
$$

This shows explicitly that $\Gamma^{\text {odd }}$ structures with a specific chirality, $P_{R}$, can indeed be generated. They do not vanish as $\lambda_{b b h} \rightarrow 0$. The even one-loop structures on the other hand are $\mathcal{O}\left(\lambda_{b}\right)$. The structure in class (c), Higgs radiation off the charged Goldstones, is the same. For class (a), radiation off the $b$ quark, the structure of the correction is different; the odd part is suppressed and receives an $\mathcal{O}\left(\lambda_{b}\right)$ correction. To summarize, with $m_{b}=0$, making explicit the Yukawa couplings and the chiral structure if any, for example $P_{R}$, that characterize each class and comparing to the leading order, one has

\begin{tabular}{lll} 
& $\Gamma^{\text {even }}$ & $\Gamma^{\text {odd }}$ \\
\hline Tree-level & $\lambda_{b b H}$ & 0 \\
(a) & $\lambda_{t}^{2} \lambda_{b b H}$ & $\lambda_{b} \lambda_{t} \lambda_{b b H}$ \\
(b) & $\lambda_{b} \lambda_{t} \lambda_{t t H}$ & $\lambda_{t}^{2} \lambda_{t t H},\left(P_{R}\right)$ \\
(c) & $\lambda_{b} \lambda_{t} \lambda_{\chi \chi H}$ & $\lambda_{t}^{2} \lambda_{\chi \chi H},\left(P_{R}\right)$
\end{tabular}

We have kept $\lambda_{f f H}$ and $\lambda_{f}$ separate to show how the structures may change in the MSSM, for example, and also why just by inspecting the couplings we can differentiate between the three classes. We clearly see that all one-loop $\Gamma^{\mathrm{even}}$ contributions vanish in the limit $\lambda_{b}=0$ or $\lambda_{b b H}=0$. On the other hand this is not the case for the one-loop $\Gamma^{\text {odd }}$ contribution belonging to classes (b) and (c). However, for these contributions to interfere with the tree-level LO contribution requires a chirality flip through a $m_{b}$ insertion. Therefore in the SM, for example, the NLO cross section is necessarily of order $m_{b}^{2}$, like the LO, with corrections proportional to the top Yukawa coupling, for example. On the other hand, in the limit of $\lambda_{b b H}=0$, the tree-level vanishes but $g g \rightarrow b \bar{b} H$ still goes with an amplitude of order $g_{s}^{2} \lambda_{t}^{2} \lambda_{t t H}$ or $g_{s}^{2} \lambda_{t}^{2} \lambda_{\chi \chi H}$. For $\lambda_{b b H} \neq 0$ these contributions should be considered as part of the NNLO "corrections"; however, they do not vanish in the limit $m_{b} \rightarrow 0$ (or $\lambda_{b b H}=0$ ) while the tree-level does. These contributions can be important and we will therefore study their effects. For these contributions at the "NNLO" we can set $m_{b}=0$.

The classification in terms of structures as we have done makes clear also that the novel one-loop induced $\Gamma^{\text {odd }}$ contributions must be ultraviolet finite. This is not necessarily the case of the $\Gamma^{\mathrm{even}}$ structures where counterterms to the tree-level structures are needed through renormalization to which we now turn.

\section{RENORMALIZATION}

We use an on-shell renormalization scheme exactly along the lines described in [15]. Ultraviolet divergences are regularized through dimensional regularization. In our approximation we only need to renormalize the vertices $b \bar{b} g$ and $b \bar{b} H$ as well as the bottom mass $m_{b}$. For the $b \bar{b} g$ vertex, from the point of view of the corrections we are carrying, only wave function renormalization for the $b / \bar{b}$ field is required: $\stackrel{(-)}{b}_{L, R} \rightarrow\left(1+\delta Z_{b_{L, R}}^{1 / 2}\right) \stackrel{(-)}{b_{L, R}} . \delta Z_{b_{L, R}}^{1 / 2}$ can be taken real; see [15]. The counterterm to $m_{b}, \delta m_{b}$, and the wave function renormalization for the $b / \bar{b}$ are set by imposing the usual conditions for pole position and residue on the renormalized bottom propagator. In terms of the self-energy correction $\Sigma_{b b}(q)$ with momentum $q$ [15]:

$$
\Sigma\left(q^{2}\right)=K_{1}+K_{\gamma} \not 1+K_{5 \gamma} \not \gamma \gamma_{5} .
$$

This translates into

$$
\begin{aligned}
\delta m_{b}= & \operatorname{Re}\left(m_{b} K_{\gamma}\left(m_{b}^{2}\right)+K_{1}\left(m_{b}^{2}\right)\right), \\
\delta Z_{b_{L}}^{1 / 2}= & \frac{1}{2} \operatorname{Re}\left(K_{5 \gamma}\left(m_{b}^{2}\right)-K_{\gamma}\left(m_{b}^{2}\right)\right) \\
& -\left.m_{b} \frac{d}{d q^{2}}\left(m_{b} \operatorname{Re} K_{\gamma}\left(q^{2}\right)+\operatorname{Re} K_{1}\left(q^{2}\right)\right)\right|_{q^{2}=m_{b}^{2}}, \\
\delta Z_{b_{R}}^{1 / 2}= & -\frac{1}{2} \operatorname{Re}\left(K_{5 \gamma}\left(m_{b}^{2}\right)+K_{\gamma}\left(m_{b}^{2}\right)\right) \\
& -\left.m_{b} \frac{d}{d q^{2}}\left(m_{b} \operatorname{Re} K_{\gamma}\left(q^{2}\right)+\operatorname{Re} K_{1}\left(q^{2}\right)\right)\right|_{q^{2}=m_{b}^{2}} .
\end{aligned}
$$

We calculate the coefficients $K_{1, \gamma, 5 \gamma}$ of the bottom selfenergy in the same spirit we calculate the other one-loop corrections, i.e., only through the $t \rightarrow b \chi_{W}$ transition; see the first diagram of class (a) in Fig. 4. We get

$$
\begin{aligned}
K_{1}\left(q^{2}\right) & =-\frac{\lambda_{t}^{2}}{16 \pi^{2}}\left(C_{\mathrm{UV}}-F_{0}\left(m_{t}, M_{W}, q^{2}\right)\right), \\
K_{\gamma}\left(q^{2}\right)= & -K_{5 \gamma}\left(q^{2}\right) \\
= & \frac{\lambda_{t}^{2}}{64 \pi^{2}}\left(C_{\mathrm{UV}}-2 F_{1}\left(m_{t}, M_{W}, q^{2}\right)\right) \\
\text { with } C_{\mathrm{UV}}= & \frac{1}{\epsilon}-\gamma_{E}+\ln 4 \pi, \\
D= & 4-2 \epsilon, \\
F_{n}\left(m_{1}, m_{2}, q^{2}\right)= & \int_{0}^{1} d x x^{n} \ln \left((1-x) m_{1}^{2}+x m_{2}^{2}\right. \\
& \left.-x(1-x) q^{2}\right) .
\end{aligned}
$$

The reason we get $K_{\gamma}\left(q^{2}\right)=-K_{5 \gamma}\left(q^{2}\right)$ is due to the particular chiral structure of the $t \rightarrow b \chi_{W}$ loop insertion. In particular, for $m_{b}=0$, one recovers that these corrections only contribute to $\delta Z_{b_{L}}^{1 / 2}$ and not $\delta Z_{b_{R}}^{1 / 2}$.

The counterterms needed to renormalize the $b \bar{b} H$ vertex are $\delta m_{b}, \delta Z_{b_{L, R}}^{1 / 2}$, as well as the Higgs wave function renormalization $\delta Z_{H}^{1 / 2}$ and the counterterm to the vacuum 
expectation value, $v, \delta v$. Indeed we have $\delta_{b b H}=$ $\lambda_{b b H}\left(\frac{\delta m_{b}}{m_{b}}+\delta Z_{b_{L}}^{1 / 2}+\delta Z_{b_{R}}^{1 / 2}+\left(\delta Z_{H}^{1 / 2}-\delta v\right)\right)$. The $t \rightarrow$ $b \chi_{W}$ loop insertion does not contribute to $\delta Z_{H}^{1 / 2}$ (which originates from the Higgs self-energy two-point function) nor to $\delta v$, the renormalization of the vacuum expectation value. On the other hand, $\left(\delta Z_{H}^{1 / 2}-\delta v\right)$ can be seen as a universal correction to Higgs production processes. We will include this correction as it has potentially large contributions scaling like $\lambda_{t}^{2}$ and $\lambda$ which fall into the category of the corrections we are seeking. Within the calculation we have performed this means that the combination $\left(\delta Z_{H}^{1 / 2}-\delta v\right)$ must be finite. Indeed, we find

$$
\begin{aligned}
\delta Z_{H}^{1 / 2}= & -\frac{1}{8 \pi^{2}} \operatorname{Re}\left\{\frac{3 \lambda_{t}^{2}}{4}\left(C_{\mathrm{UV}}-F_{0}\left(m_{t}, m_{t}, M_{H}^{2}\right)-M_{H}^{2} G_{0}\left(m_{t}, m_{t}, M_{H}^{2}\right)+4 m_{t}^{2} G_{0}\left(m_{t}, m_{t}, M_{H}^{2}\right)\right)\right. \\
& \left.-\frac{\lambda}{4}\left(9 G_{0}\left(M_{H}, M_{H}, M_{H}^{2}\right)+2 G_{0}\left(M_{W}, M_{W}, M_{H}^{2}\right)+G_{0}\left(M_{Z}, M_{Z}, M_{H}^{2}\right)\right)\right\} \\
\delta v= & -\frac{1}{8 \pi^{2}} \operatorname{Re}\left\{\frac{3 \lambda_{t}^{2}}{4}\left(C_{\mathrm{UV}}-2 F_{1}\left(m_{b}, m_{t}, M_{W}^{2}\right)\right)-\lambda\left(F_{0}\left(M_{H}, M_{W}, M_{W}^{2}\right)-F_{1}\left(M_{H}, M_{W}, M_{W}^{2}\right)-\frac{1}{2} \ln M_{H}^{2}\right)\right. \\
& -\frac{c_{W}^{2}}{s_{W}^{2}}\left(\frac{3 \lambda_{t}^{2}}{4}\left(F_{0}\left(m_{t}, m_{t}, M_{Z}^{2}\right)-2 F_{1}\left(m_{b}, m_{t}, M_{W}^{2}\right)\right)+\lambda\left(F_{0}\left(M_{H}, M_{Z}, M_{Z}^{2}\right)-F_{1}\left(M_{H}, M_{Z}, M_{Z}^{2}\right)\right.\right. \\
& \left.\left.\left.-F_{0}\left(M_{H}, M_{W}, M_{W}^{2}\right)+F_{1}\left(M_{H}, M_{W}, M_{W}^{2}\right)\right)\right)\right\} \\
G_{n}\left(m_{1}, m_{2}, q^{2}\right)= & q^{2} \frac{d}{d q^{2}} F_{n}\left(m_{1}, m_{2}, q^{2}\right)=q^{2} \int_{0}^{1} d x \frac{-x^{n} x(1-x)}{(1-x) m_{1}^{2}+x m_{2}^{2}-x(1-x) q^{2}}
\end{aligned}
$$

which shows that $\left(\delta Z_{H}^{1 / 2}-\delta v\right)$ is finite.

In the actual calculation, the counterterm $\delta_{b b g}^{\mu}$ belongs to class (a) in the classification of Fig. 4. This makes class (a) finite. The counterterm we associate to class (b) is the part of $\delta_{b b H}$ from the $t \rightarrow b \chi_{W}$ loops and therefore does not include what we termed the universal Higgs correction, i.e., does not include the contribution $\left(\delta Z_{H}^{1 / 2}-\right.$ $\delta v$ ). This is sufficient to make class (b) finite. In our approach (c) is finite without the addition of a counterterm. We will keep the $\left(\delta Z_{H}^{1 / 2}-\delta v\right)$ contribution separate from the contributions in classes (a), (b), (c). We will of course include it in the final result.

\section{CALCULATION DETAILS}

We have written two independent codes. In the first one we set $m_{b}=0$ in all propagators and other spinors that emerge from the helicity formalism we follow. In this limit, the helicity formalism is very much simplified and the expression quite compact. This code is in fact subdivided in two separate subcodes. One subcode is generated for the "even" part [constituted by the $\Gamma^{\mathrm{even}}$ contributions, see Eq. (2)] and the other by the "odd" part. We also generate a completely independent code for the case $m_{b} \neq 0$ where, in particular, we use the helicity formalism with massive fermions. Details of the helicity formalism that we use are given in Appendix A.

The steps that go into writing these codes are the following. In the first stage, we use FORM [16] to generate expressions for the tree-level and one-loop helicity amplitudes. Each helicity amplitude is written in terms of
Lorentz invariants, scalar spinor functions $(A, B, C)_{\lambda_{i} \lambda_{j}}$ defined in Appendix A, and the Passarino-Veltman [17] tensor functions $T_{M}^{N}$ for a tensor of rank $M$ for $N$-point function. We have also sought to write the contribution of each amplitude as a product of different structures or blocks that reappear for different graphs and contributions. For example, color factorization is implemented; this further allows us to rearrange the amplitude into an Abelian part and a non-Abelian part which will not interfere with each other at the matrix element squared level. The helicity information is contained in a set of basic blocks for further optimization. Another set of blocks pertains to the loop integrals and other elements. The factorization of the full amplitude in terms of independent building blocks is easily processed within FORM. These building blocks can still consist of long algebraic expressions which can be efficiently abbreviated into compact variables with the help of a PERL script which also allows us to convert the output of FORM into the FORTRAN code ready for a numerical evaluation. More details on the connection between FORM and FORTRAN as well as the optimization we implemented in the codes can be found in Appendix B.

\section{A. Loop integrals, Gram determinants, and phase-space integrals}

The highest rank $M$ of the Passarino-Veltman tensor functions $T_{M}^{N}$ with $M \leq N$ that we encounter in our calculation is $M=4$ and is associated to a pentagon graph, $N=$ 5. We use the library LOOPTOOLS [18] to calculate all the tensorial one-loop integrals as well as the scalar integrals, 
this means that we leave it completely to LOOPTOOLS to perform the reduction of the tensor integrals to the basis of the scalar integrals. In order to obtain the cross section one needs to perform the phase-space integration and convolution over the gluon distribution function (GDF), $g(x, Q)$ with $Q$ representing the factorization scale. We have

$$
\begin{aligned}
\sigma(p p \rightarrow b \bar{b} H)= & \frac{1}{256} \int_{0}^{1} d x_{1} g\left(x_{1}, Q\right) \int_{0}^{1} d x_{2} g\left(x_{2}, Q\right) \frac{1}{\hat{F}} \\
& \times \int \frac{d^{3} \mathbf{p}_{3}}{2 e_{3}} \frac{d^{3} \mathbf{p}_{4}}{2 e_{4}} \frac{d^{3} \mathbf{p}_{5}}{2 e_{5}} \\
& \times|\mathcal{A}(g g \rightarrow b \bar{b} H)|^{2} \\
& \times \delta^{4}\left(p_{1}+p_{2}-p_{3}-p_{4}-p_{5}\right),
\end{aligned}
$$

where $\frac{1}{256}=\frac{1}{4} \times \frac{1}{8} \times \frac{1}{8}$ is the spin and color average factor and the flux factor is $1 / \hat{F}=1 /\left((2 \pi)^{5} 2 \hat{s}\right)$ with $\hat{s}=$ $x_{1} x_{2} s \geq\left(2 m_{b}+M_{H}\right)^{2}$.

The integration over the three body phase space and momentum fractions of the two initial gluons is done by using two "integrators": BASES [19] and DADMUL [20]. BASES is a Monte Carlo that uses the importance sampling technique while DADMUL is based on the adaptive quadrature algorithm. The use of two different phase-space integration routines helps control the accuracy of the results and helps detect possible instabilities. In fact, some numerical instabilities in the phase-space integration do occur when we use DADMUL but not when we use BASES, which gives very stable results with small integration error, typically $0.08 \%$ for $10^{5}$ Monte Carlo points. For the range of Higgs masses we are studying in this paper, the instabilities that are detected with DADMUL were identified as spurious singularities having to do with vanishing Gram determinants for the three and four point tensorial functions calculated in LOOPTOOLS by using the PassarinoVeltman reduction method. ${ }^{2}$ Because this problem always happens at the boundary of phase space, we can avoid it by imposing appropriate kinematic cuts in the final state. In our calculation, almost all zero Gram determinants disappear when we apply the cuts on the transverse momenta of the bottom quarks relevant for our situation; see Sec. VA for the choice of cuts. The remaining zero Gram determinants occur when the two bottom quarks or one bottom quark and the Higgs are produced in the same direction. Our solution, once identified as spurious, was to discard these points by imposing some tiny cuts on the polar, $\theta$, and relative azimuthal angles, $\phi$ of the outgoing $b$ quarks, the value of the cuts is $\theta_{\text {cut }}^{b, \bar{b}}=\left|\sin \phi^{\bar{b}}\right|_{\text {cut }}=10^{-6}$. DADMUL then produces the same result as BASES within the integration error.

\footnotetext{
${ }^{2}$ The reduction of the five point function using the method of Denner and Dittmaier [21] and Hahn and Rauch [22] which avoids the Gram determinant at this stage as implemented in LOOPTOOLS gives very stable results.
}

\section{B. Checks on the results}

i) Ultraviolet finiteness: The final results must be ultraviolet finite. It means that they should be independent of the parameter $C_{\mathrm{UV}}$ defined in Eq. (8). In our code this parameter is treated as a variable. The cancellation of $C_{\mathrm{UV}}$ has been carefully checked in our code. Upon varying the value of the parameter $C_{\mathrm{UV}}$ from $C_{\mathrm{UV}}=0$ to $C_{\mathrm{UV}}=$ $10^{5}$, the result is stable within more than 9 digits using double precision. This check makes sure that the divergent part of the calculation is correct. The correctness of the finite part is also well checked in our code by confirming that each helicity configuration is QCD gauge invariant.

ii) QCD gauge invariance: In the physical gauge we use, the QCD gauge invariance reflects the fact that the gluon is massless and has only two transverse polarization components. In the helicity formalism that we use, the polarization vector of the gluon of momentum $p$ and helicity $\lambda$ is constructed with the help of a reference vector $q$; see Appendix A for details. The polarization vector is then labeled as $\varepsilon^{\mu}(p, \lambda ; q)$. A change of reference vector from $q$ to $q^{\prime}$ amounts essentially to a gauge transformation (up to a phase)

$$
\varepsilon^{\mu}\left(p, \lambda ; q^{\prime}\right)=e^{i \phi\left(q^{\prime}, q\right)} \varepsilon^{\mu}(p, \lambda ; q)+\beta\left(q^{\prime}, q\right) p^{\mu} .
$$

QCD gauge invariance in our case amounts to independence of the cross section in the choice of the reference vector $q$. We have carefully checked that the numerical result for the norm of each helicity amplitude at various points in phase space is independent of the reference vectors, say $q_{1,2}$ for gluon 1 and 2, up to 12 digits using double precision. By default, our numerical evaluation is based on the use of $q_{1,2}=\left(p_{2}, p_{1}\right)$. For the checks in the case of massive $b$ quarks the result with the default choice $q_{1,2}=\left(p_{2}, p_{1}\right)$ is compared with a random choice of $q_{1,2}$, keeping away from vectors with excessively too small or too large components; see Appendix A for more details.

iii) As stated earlier, the results based on the use of the massive quark helicity amplitude are checked against those with the independent code using the massless helicity amplitude by setting the mass of the $b$ quark to zero. This is however just a consistency check.

iv) At the level of integration over phase space and density functions we have used two integration routines and made sure that we obtain the same result once we have properly dealt with the spurious Gram determinant as we explained in Sec. IVA.

v) Moreover, our tree-level results have been successfully checked against the results of CALCHEP [23].

\section{RESULTS}

\section{A. Input parameters and kinematical cuts}

Our input parameters are $\alpha(0)=1 / 137.03599911$, $M_{W}=80.3766 \mathrm{GeV}, \quad M_{Z}=91.1876 \mathrm{GeV}, \quad \alpha_{s}\left(M_{Z}\right)=$ $0.118, m_{b}=4.62 \mathrm{GeV}, m_{t}=174.0 \mathrm{GeV}$ with $s_{W} \equiv$ $\sqrt{1-M_{W}^{2} / M_{Z}^{2}}$. The Cabibbo-Kobayashi-Maskawa 
(CKM) parameter $V_{t b}$ is set to be 1 . We consider the case at the LHC where the center of mass energy of the two initial protons is $\sqrt{s}=14 \mathrm{TeV}$. Neglecting the small light quark initiated contribution, we use CTEQ6L [24] for the GDF in the proton. The factorization scale for the GDF and energy scale for the strong coupling constant are chosen to be $Q=$ $M_{Z}$ for simplicity.

As has been done in previous analyses [5,25], for the exclusive $b \bar{b} H$ final state, we require the outgoing $b$ and $\bar{b}$ to have high transverse momenta $\left|\mathbf{p}_{T}^{b, \bar{b}}\right| \geq 20 \mathrm{GeV}$ and pseudorapidity $\left|\eta^{b, \bar{b}}\right|<2.5$. These kinematical cuts reduce the total rate of the signal but also greatly reduce the QCD background. As pointed out in [4] these cuts also stabilize the scale dependence of the QCD NLO corrections com- pared to the case where no cut is applied. In the following, these kinematical cuts are always applied unless otherwise stated.

Speaking of the NLO QCD scale uncertainty and before presenting our results, let us remind the reader of the size of the QCD corrections. Taking a renormalization/factorization scale as we take here at $M_{Z}$, the QCD corrections in a scheme where the bottom Yukawa coupling is taken on shell amount to $\sim-22 \%$ for a Higgs mass of $120 \mathrm{GeV}$.

\section{B. NLO EW correction with $\lambda_{b b H} \neq 0$}

The cross sections with two high- $p_{T}$ bottom quarks at $\mathrm{LO}$ and NLO at the LHC are displayed in Fig. 5 as a function of the Higgs mass. The NLO EW correction
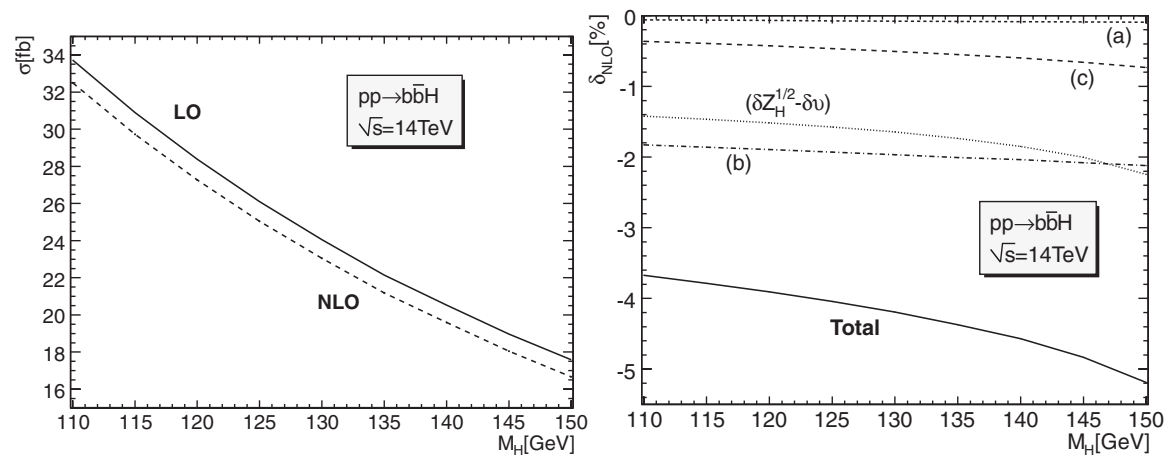

FIG. 5. Left: The LO and NLO cross sections as functions of $M_{H}$. Right: The relative NLO EW correction normalized to tree-level $\sigma_{\mathrm{LO}}(\mathrm{a}),(\mathrm{b})$, and (c) correspond to the three classes of diagrams as displayed in Fig. 4 to which counterterms are added (see Sec. III). $\left(\delta Z_{H}^{1 / 2}-\delta v\right)$ is the correction due to the universal correction contained in the renormalization of the $b \bar{b} H$ vertex. "Total" refers to the total electroweak correction, of Yukawa type, at one-loop.
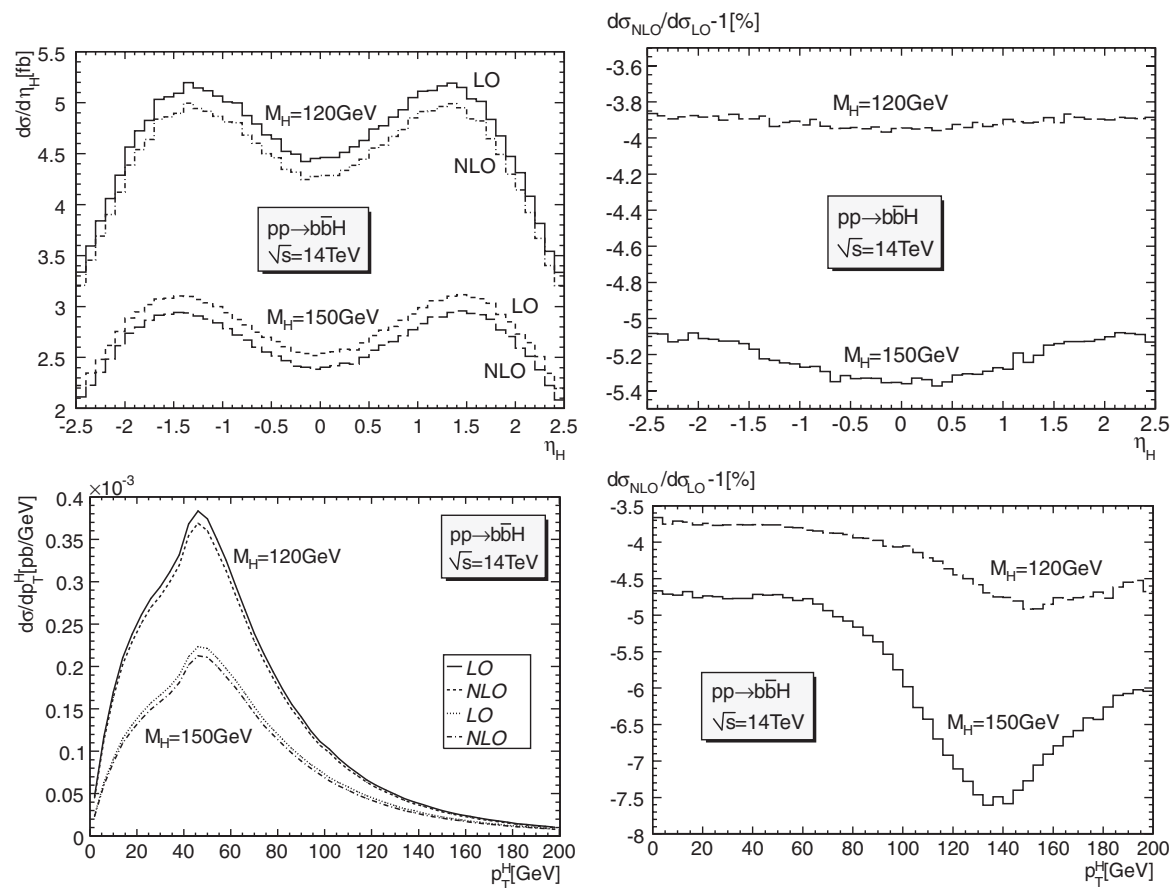

FIG. 6. Effect of the NLO electroweak corrections on the pseudorapidity and transverse momentum distributions of the Higgs for $M_{H}=120,150 \mathrm{GeV}$. The relative correction $d \sigma_{\mathrm{NLO}} / d \sigma_{\mathrm{LO}}-1$ is also shown. 

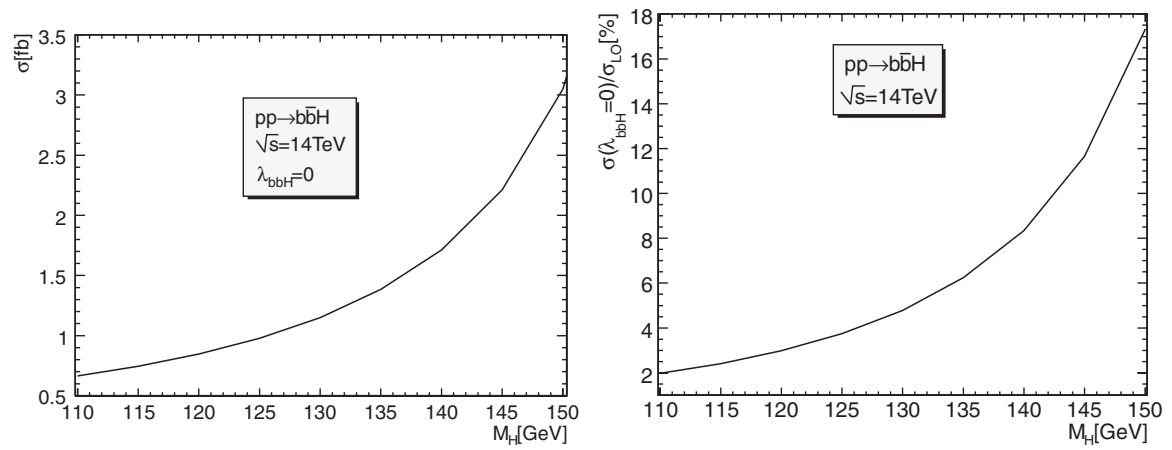

FIG. 7. The one-loop induced cross section as a function of $M_{H}$ in the limit of vanishing bottom-Higgs Yukawa coupling. The right panel shows the percentage contribution of this contribution relative to the tree-level cross section calculated with $\lambda_{b b H} \neq 0$.

reduces the cross section by about $4 \%$ to $5 \%$ as the Higgs mass is varied from 110 to $150 \mathrm{GeV}$. The first conclusion to draw is that this correction is small if we compare it to the QCD correction or even to the QCD scale uncertainty.
Considering that we have pointed to the fact that the contributions could be grouped into three gauge invariant classes that reflect the strengths of the Higgs coupling to the $b$, the $t$, or its self-coupling, one can ask whether this is
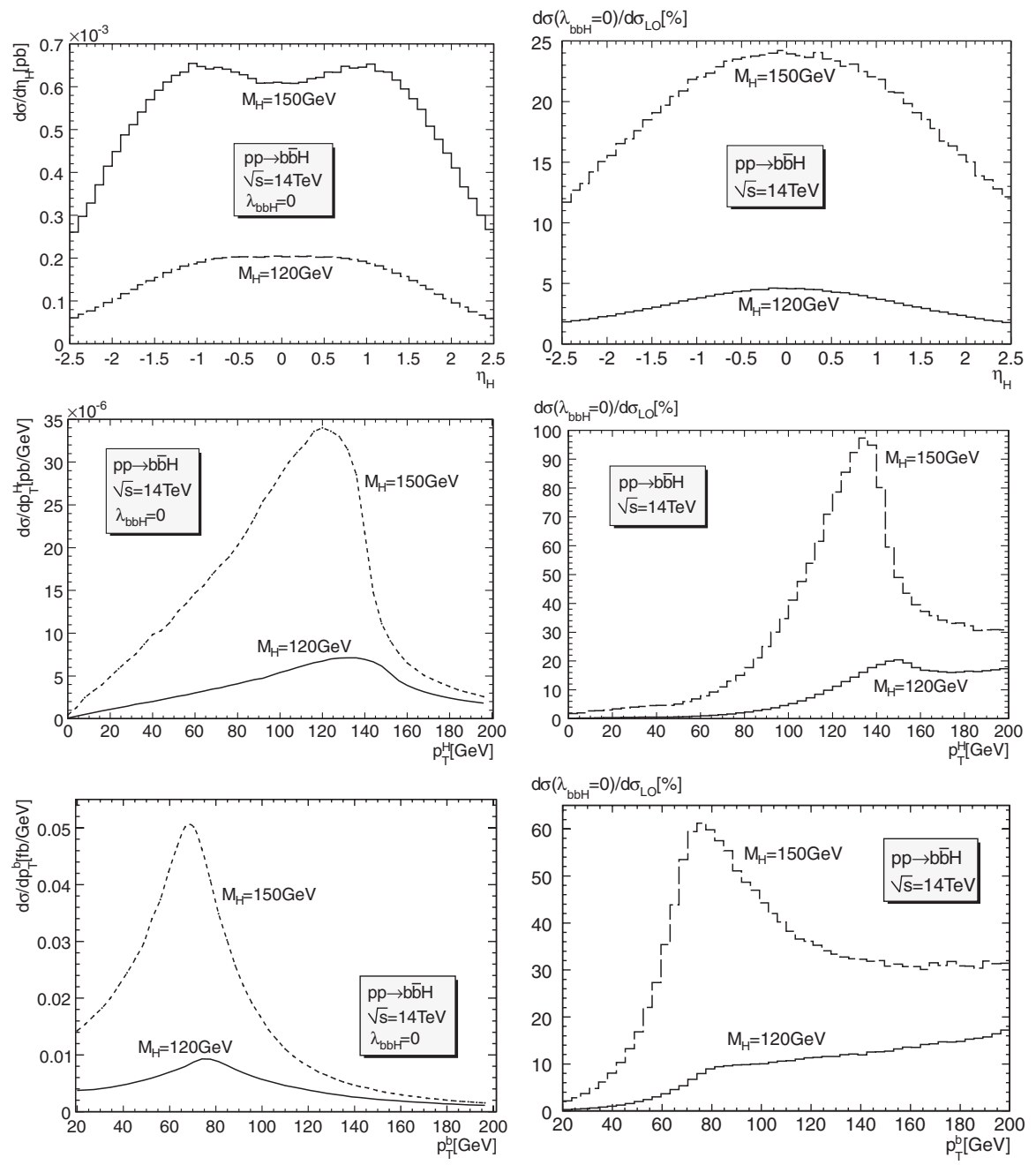

FIG. 8. The pseudorapidity of the Higgs and transverse momentum distributions of the Higgs and the bottom for $M_{H}=$ $120,150 \mathrm{GeV}$ arising from the purely one-loop contribution in the limit of vanishing $\mathrm{LO}\left(\lambda_{b b H}=0\right)$. Its relative percentage contribution $d \sigma\left(\lambda_{b b H}=0\right) / d \sigma_{\mathrm{LO}}$ is also shown. 
the result of some cancellation. It turns out not to be the case. All contributions are below 3\%; see Fig. 5. Class (a) with a Higgs radiated from the bottom line is totally negligible ranging from $-0.09 \%$ to $-0.06 \%$. We have failed in finding a good reason for the smallness of this contribution compared to the others. Those due to the Higgs self-coupling are below 1\%. Radiation from the top contributes about $-2 \%$ and is of the same order as the contribution of the universal correction. We had argued that the Yukawa corrections brought about by the top might be large. It seems that the mass of the top introduces also a large scale which cannot be neglected compared to the effective energy of the hard process even for LHC energies.

The NLO corrections are spread rather uniformly on all the distributions we have looked at. We have chosen to show in Fig. 6 the effect on pseudorapidity and transverse momentum distributions of the Higgs for two cases $M_{H}=$ $120 \mathrm{GeV}$ and $M_{H}=150 \mathrm{GeV}$. As Fig. 6 shows, the relative change in these two distributions is sensibly constant especially for $M_{H}=120 \mathrm{GeV}$. For $M_{H}=150 \mathrm{GeV}$, the corrections are largest for $p_{T}^{H}$ around $140 \mathrm{GeV}$; however, this is where the cross section is very small. A similar pattern, i.e., a constant change in the distributions, is observed for the bottom variables.

\section{EW correction in the limit of vanishing $\lambda_{b b H}$}

The cross section for $\lambda_{b b H}=0$ can be induced at oneloop through the top loop. This NNLO contribution rises rather quickly as the Higgs mass increases even in the narrow range $M_{H}=110-150 \mathrm{GeV}$, as can be seen in Fig. 7. Indeed relative to the tree-level, the cross section with $M_{H}=120 \mathrm{GeV}$ amounts to $3 \%$ while for $M_{H}=$ $150 \mathrm{GeV}$ it has increased to as much as $17 \%$. Going past $M_{H} \geq 2 M_{W}$ we encounter a Landau singularity [26] (a pinch singularity in the loop integral) from diagrams like the one depicted in Fig. 2 (right) with the Higgs being attached to the $W$ 's or their Goldstone counterpart. It corresponds to a situation where all particles in the loop are resonating and can be interpreted as the production and decay of the tops into (longitudinal) W's with the later fusing to produce the Higgs. This leading Landau singularity is not integrable, at the level of the loop amplitude squared, and must be regulated by the introduction of a width for the unstable particles. We leave this issue together with a general discussion of Landau singularities in such situations to another publication.

Figure 8 shows the pseudorapidity and transverse momentum distributions of the Higgs as well as the $p_{T}$ of the bottom for two cases $M_{H}=120 \mathrm{GeV}$ and $M_{H}=150 \mathrm{GeV}$ in the limit of vanishing bottom-Higgs Yukawa coupling. These distributions are significantly different from the ones we observed at tree-level (and with the electroweak NLO corrections); see Fig. 6. The Higgs prefers being produced at high value of transverse momentum, about $130 \mathrm{GeV}$. In the case of a Higgs with $M_{H}=150 \mathrm{GeV}$ this contribution can significantly distort the shape of the $p_{T}^{H}$ distribution for high $p_{T}^{H}$ with a "correction" of more than $70 \%$ over a rather large range. The distribution in the $p_{T}$ of the bottom is also very telling. The new contributions do not produce the bottom preferentially with low $p_{T}^{b}$ as the case of the LO contribution.

\section{CONCLUSIONS}

We have calculated the EW radiative corrections triggered by the Yukawa coupling of the top to the process $p p \rightarrow b \bar{b} H$ at the LHC through gluon fusion in the SM. This process is triggered through Higgs radiation of the bottom quark with a small coupling proportional to the mass of the bottom. Yet in order to analyze this coupling, precision calculations that include both the QCD and electroweak corrections are needed. In this perspective, to identify the process one needs to tag both $b$ jets. Our calculation is therefore conducted in this kinematical configuration. Inserting a top quark loop with a Yukawa transition of the type $t \rightarrow b \chi_{W}, \chi_{W}$ is the charged Goldstone, allows now the Higgs to be radiated from the top or from the Goldstone boson. The latter coupling represents the Higgs self-coupling and increases with the Higgs mass. The former, the top Yukawa coupling, is also large. As a consequence, the one-loop amplitude $g g \rightarrow b \bar{b} H$ no longer vanishes as the Higgs coupling to $b$ 's does, like what occurs at leading order. We find that in the limit of vanishing $\lambda_{b b H}$, the one-loop induced electroweak process should be taken into account for Higgs masses larger than $140 \mathrm{GeV}$ or so. Indeed, though this contribution is quite modest for a Higgs mass of $110 \mathrm{GeV}$ it increases quite rapidly as the Higgs mass increases, reaching about $17 \%$ of the leading order value, calculated with $m_{b}=4.62 \mathrm{GeV}$, for $M_{H}=150 \mathrm{GeV}$. For these new corrections to interfere with the leading order requires helicity flip. Therefore at next-to-leading order in the Yukawa electroweak corrections, all corrections involve either a bottom mass insertion or a bottom Yukawa coupling. At the end the total Yukawa electroweak NLO contribution brings in a correction which is within the range $-4 \%$ to $-5 \%$ for Higgs masses in the range $110<M_{H}<150 \mathrm{GeV}$. They are therefore negligible compared to the NLO QCD correction and even the remaining QCD scale uncertainty. This modest effect translates also as a uniform rescaling of the distributions in the most interesting kinematical variables we have looked at (pseudorapidities and $p_{T}$ of both $b$ quarks and the Higgs). This is not the case of the one-loop induced contributions which survive in the limit of $m_{b} \rightarrow 0$ (and $\lambda_{b b H} \rightarrow 0$ ). Here the distributions for the Higgs masses where the corrections for the total cross section are large are drastically different from the LO distributions. A summary for the corrections including the NLO with $\lambda_{b b H} \neq 0$ and the part of the NNLO counted as loop induced in the limit $\lambda_{b b H} \rightarrow 0$ is shown in Fig. 9. 


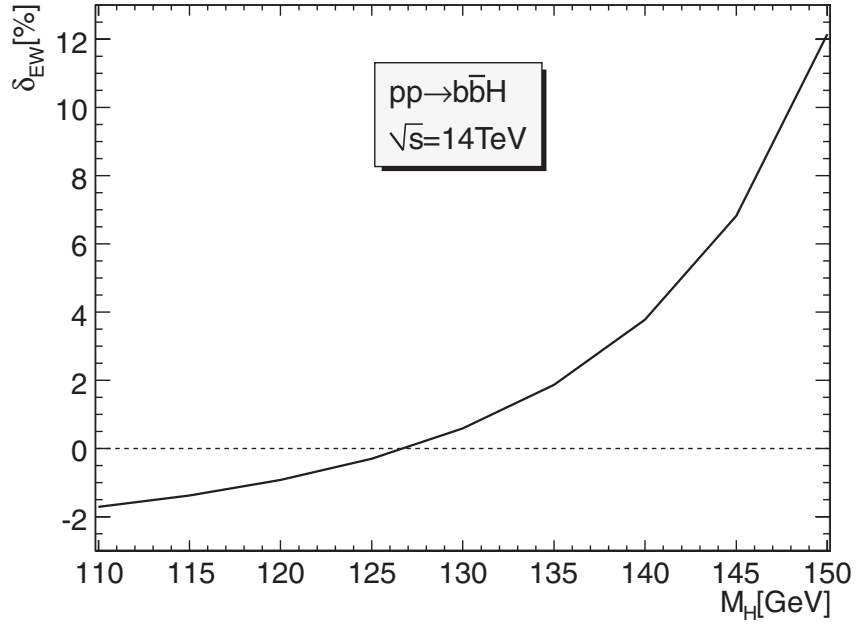

FIG. 9. $\delta_{\mathrm{EW}}=\delta_{\mathrm{NLO}}+\frac{\sigma\left(\lambda_{b b H}=0\right)}{\sigma_{0}}$ as a function of $M_{H}$.

The analysis we have performed in this paper does not cover Higgs masses over $150 \mathrm{GeV}$ and rests within the range of Higgs masses preferred by indirect precision measurements. In fact as the threshold for $H \rightarrow W W$ opens up, important phenomena take place. Foremost a Landau singularity, or a pinch singularity in some loop integrals, develops. This corresponds to the rescattering of on-shell top quarks that decay to on-shell $W$ with Higgs production via $W W$ fusion. We leave this important issue to a forthcoming publication especially that the identification and handling of such singularities can be applied to other processes. In our case the singularity can be tamed by introducing the width of the unstable particles. At NLO, for $M_{H}=2 M_{W}$ for example, the wave function renormalization of the Higgs, which involves the derivative of the two-point function Higgs self-energy, diverges. This can also be regulated by including the width of the $W$; see for example [27].

There is another contribution which does not vanish for vanishing $\lambda_{b b H}$ and which contributes to $g g \rightarrow b \bar{b} H$ through a closed top quark loop. This contribution represents $g g \rightarrow H g^{*} \rightarrow H b \bar{b}$. We have not included this contribution in the present paper as we do not consider it to be a genuine $b \bar{b} H$ final state. This correction can be counted as belonging to the inclusive $g g \rightarrow H$ process. The same line of reasoning has been argued in [7]. Nonetheless, from the experimental point of view it would be interesting to include all these effects together with the NLO QCD corrections and the electroweak corrections that we have studied here.

\section{ACKNOWLEDGMENTS}

L. D. N. expresses his gratitude and thanks to G. Altarelli and P. Aurenche for their supervision, support, and most helpful discussions and comments. We benefited a lot from discussions with J. P. Guillet and P. Slavich. We also acknowledge discussions with G. Bélanger, Dao Thi Nhung,
Do Hoang Son, J. Ellis, J. Fujimoto, K. Kato, Y. Kurihara, M. Mühlleitner, E. Pilon, P. Uwer, and J. Vermaseren. L.D.N. acknowledges the financial support of Rencontres du Vietnam and the Marie Curie Early Stage Training Grant of the European Commission.

\section{APPENDIX A: THE HELICITY AMPLITUDE METHOD}

\section{Method}

We use a combination of helicity amplitude methods as described in $[28,29]$ to calculate the total cross section. In the following we only want to highlight some key features that were most useful for our calculation; for details of the method we refer to [28,29]. For our process $g\left(p_{1}, \lambda_{1}\right)+$ $g\left(p_{2}, \lambda_{2}\right) \rightarrow b\left(p_{3}, \lambda_{3}\right)+\bar{b}\left(p_{4}, \lambda_{4}\right)+H\left(p_{5}\right)$ where the particles are denoted by their momentum $p_{i}$ and helicity $\lambda_{i}$ we write the corresponding helicity amplitude as $\mathcal{A}\left(\lambda_{1}, \lambda_{2} ; \lambda_{3}, \lambda_{4}\right)$.

$$
\begin{aligned}
\mathcal{A}\left(\lambda_{1}, \lambda_{2} ; \lambda_{3}, \lambda_{4}\right)= & \varepsilon_{\mu}\left(p_{1}, \lambda_{1} ; q_{1}\right) \varepsilon_{\nu}\left(p_{2}, \lambda_{2} ; q_{2}\right) \\
& \times \mathcal{M}^{\mu \nu}\left(\lambda_{3}, \lambda_{4}\right), \\
\mathcal{M}^{\mu \nu}\left(\lambda_{3}, \lambda_{4}\right)= & \bar{u}\left(p_{3}, \lambda_{3}\right) \Gamma^{\mu \nu} v\left(p_{4}, \lambda_{4}\right) .
\end{aligned}
$$

$\Gamma^{\mu \nu}$ is a string of Dirac $\gamma$ matrices. These $\gamma$ matrices represent either interaction vertices or momenta from the fermion propagators. In our case the interaction vertices are the vectorial gluon vertices in which case they represent $\xi_{i}$, the scalar Higgs vertex and at one-loop the pseudoscalar Goldstone coupling. For the momenta, in our implementation we reexpress them in terms of the independent external momenta $p_{1}, p_{2}, p_{3}, p_{4}$. This applies also to the loop momenta after the reduction formalism of the tensor integrals has been performed. The first step in the idea of the helicity formalism we follow is to turn each of these $\gamma$ matrices (apart from the pseudoscalar and the trivial scalar) into a combination of spinor function $u \bar{u}$. We therefore transform our helicity amplitude into products of spinors such as the helicity amplitude could be written like a product $\bar{u} u \bar{u} \ldots u \bar{u}$ with the possible insertion of $\gamma_{5}$ 's in the string. The different $u, \bar{u}, v$ in the string we have written have of course, in general, different arguments. Nonetheless one can turn each spinor product of two adjacent $\bar{u} u$, etc. into a complex number written in terms of the momenta in our problem as we will see.

In the first step, for the momentum $p_{i}$ with $p_{i}^{2}=m_{i}^{2}$ we use

$$
\not p_{i}=u\left(p_{i},-\right) \bar{u}\left(p_{i},-\right)+u\left(p_{i},+\right) \bar{u}\left(p_{i},+\right)-m_{i} .
$$

The polarization vector of the initial gluon $i, \varepsilon_{\mu}\left(p_{i}, \lambda_{i} ; q_{i}\right)$, is also first expressed in terms of spinors such as

$$
\epsilon_{\mu}\left(p_{i}, \lambda_{i} ; q_{i}\right)=\frac{\bar{u}\left(p_{i}, \lambda_{i}\right) \gamma_{\mu} u\left(q_{i}, \lambda_{i}\right)}{\left[4\left(p_{i} \cdot q_{i}\right)\right]^{1 / 2}},
$$

where $q_{i}$ is an arbitrary reference vector satisfying the 
following conditions

$$
q_{i}^{2}=0, \quad p_{i} \cdot q_{i} \neq 0 .
$$

Gauge invariance (transversality condition) requires that the cross sections are independent of the choice of the reference vector as we will see later. This acts as an important check of the calculation; see later. It is not difficult to prove that the choice (A3) satisfies all the conditions for a transverse polarization vector. In particular,

$$
\begin{gathered}
p_{i} \cdot \varepsilon\left(p_{i}, \lambda_{i}\right)=0, \quad \varepsilon\left(p_{i}, \lambda_{i}\right) \cdot \varepsilon\left(p_{i}, \lambda_{i}\right)=0, \\
\varepsilon_{\mu}\left(p_{i},-\lambda_{i}\right)=\varepsilon_{\mu}\left(p_{i}, \lambda_{i}\right)^{*}, \\
\varepsilon\left(p_{i}, \lambda_{i}\right) \cdot \varepsilon\left(p_{i},-\lambda_{i}\right)=-1,
\end{gathered}
$$

where the reference vector is not written down explicitly. $i=1,2$ and no sum over $i$ must be understood. Then for $\xi_{i}=\epsilon_{\mu} \gamma^{\mu}$ one uses the so-called Chisholm identity

$$
\begin{aligned}
\bar{u}(p, \lambda) \gamma_{\mu} u(q, \lambda) \gamma^{\mu}= & 2[u(p,-\lambda) \bar{u}(q,-\lambda) \\
& +u(q, \lambda) \bar{u}(p, \lambda)],
\end{aligned}
$$

where all the spinors in Eq. (A6) are for massless states in view of the lightlike condition on the reference frame vector and of course the momentum of the real gluon.

With $U\left(p_{i}, \lambda_{i}\right)$ representing either $u\left(p_{i}, \lambda_{i}\right)$ or $v\left(p_{i}, \lambda_{i}\right)$ one uses the general formulas

$$
\begin{aligned}
\bar{U}\left(p_{i}, \lambda_{i}\right) U\left(p_{j}, \lambda_{j}\right) & =\frac{A_{\lambda_{i} \lambda_{j}}\left(p_{i}, p_{j}\right)+M_{i} B_{\lambda_{i} \lambda_{j}}\left(p_{i}, p_{j}\right)+M_{j} C_{\lambda_{i} \lambda_{j}}\left(p_{i}, p_{j}\right)}{\sqrt{\left(p_{i} \cdot k_{0}\right)\left(p_{j} \cdot k_{0}\right)}}, \\
\bar{U}\left(p_{i}, \lambda_{i}\right) \gamma_{5} U\left(p_{j}, \lambda_{j}\right) & =-\lambda_{i} \frac{A_{\lambda_{i} \lambda_{j}}\left(p_{i}, p_{j}\right)-M_{i} B_{\lambda_{i} \lambda_{j}}\left(p_{i}, p_{j}\right)+M_{j} C_{\lambda_{i} \lambda_{j}}\left(p_{i}, p_{j}\right)}{\sqrt{\left(p_{i} \cdot k_{0}\right)\left(p_{j} \cdot k_{0}\right)}},
\end{aligned}
$$

where

$$
\begin{aligned}
M_{i}= & +m_{i} \quad \text { if } U\left(p_{i}, \lambda_{i}\right)=u\left(p_{i}, \lambda_{i}\right), \\
M_{i}= & -m_{i} \quad \text { if } U\left(p_{i}, \lambda_{i}\right)=v\left(p_{i}, \lambda_{i}\right), \\
A_{\lambda_{i} \lambda_{j}}= & \delta_{\lambda_{i}-\lambda_{j}} \lambda_{i}\left(\left(k_{0} \cdot p_{i}\right)\left(k_{1} \cdot p_{j}\right)\right. \\
& \left.-\left(k_{0} \cdot p_{j}\right)\left(k_{1} \cdot p_{i}\right)-i \lambda_{i} \epsilon_{\mu \nu \rho \sigma} k_{0}^{\mu} k_{1}^{\nu} p_{i}^{\rho} p_{j}^{\sigma}\right), \\
B_{\lambda_{i} \lambda_{j}}= & \delta_{\lambda_{i} \lambda_{j}}\left(k_{0} \cdot p_{j}\right), C_{\lambda_{i} \lambda_{j}}=\delta_{\lambda_{i} \lambda_{j}}\left(k_{0} \cdot p_{i}\right),
\end{aligned}
$$

with $k_{0,1}$ being auxiliary vectors such that $k_{0}^{2}=0, k_{1}^{2}=$ -1 , and $k_{0} \cdot k_{1}=0$. No sum over repeated indices must be understood. For instance, we can choose $k_{0}=(1,0,1,0)$ and $k_{1}=(0,1,0,0)$. With this choice, it is obvious to see that the denominator in (A7) can never vanish if the bottom mass is kept. If one would like to neglect $m_{b}$, that choice can bring $p_{3} \cdot k_{0}$ or $p_{4} \cdot k_{0}$ to zero in some cases. If this happens, one can tell the code to choose $k_{0}=(1,0,-1,0)$ instead of the above choice. In fact, that is what we did in our codes.

In the case of spinors representing a massless state, the helicity formalism simplifies considerably. Only $A_{\lambda_{i} \lambda_{j}}$ is needed. Traditionally we introduce the $C$ numbers $s(p, q)$ and $t(p, q)$,

$$
\begin{aligned}
& s(p, q) \equiv \bar{u}(p,+) u(q,-)=A_{+-}(p, q), \\
& t(p, q) \equiv \bar{u}(p,-) u(q,+)=-s(p, q)^{*} .
\end{aligned}
$$

These are the functions that appear in our code for the massless $b$ quark. The massless case is also used when expressing the gluon polarization vector to which we now turn.

\section{Transversality and gauge invariance}

The reference vector used for the polarization of the gluon can be changed at will. Changing the reference vector from $q$ to $q^{\prime}$ amounts essentially to a gauge transformation. Indeed one has [28]

$$
\varepsilon^{\mu}\left(p, \lambda ; q^{\prime}\right)=e^{i \phi\left(q^{\prime}, q\right)} \varepsilon^{\mu}(p, \lambda ; q)+\beta\left(q^{\prime}, q\right) p^{\mu},
$$

where

$$
\begin{aligned}
& e^{i \phi\left(q^{\prime}, q\right)}=\left[\frac{s(p, q)}{t(p, q)} \frac{t\left(p, q^{\prime}\right)}{s\left(p, q^{\prime}\right)}\right]^{1 / 2}, \\
& \beta\left(q^{\prime}, q\right)=\frac{2}{\left[4\left(q^{\prime} \cdot p\right)\right]^{1 / 2}} \frac{t\left(q, q^{\prime}\right)}{t(q, p)} .
\end{aligned}
$$

Therefore up to the phase factor, the difference is contained in the momentum vector of the gluon. QCD gauge invariance for our process leads to the important identity

$$
\left|\mathcal{A}\left(\lambda_{1}, \lambda_{2} ; \lambda_{3}, \lambda_{4} ; q_{1}, q_{2}\right)\right|^{2}=\left|\mathcal{A}\left(\lambda_{1}, \lambda_{2} ; \lambda_{3}, \lambda_{4} ; q_{1}^{\prime}, q_{2}^{\prime}\right)\right|^{2},
$$

as long as $q_{1,2}^{\prime}$ satisfy the condition (A4). We have carefully checked that the numerical result for the norm of each helicity amplitude at various points in phase space is independent of the reference vectors $q_{1,2}$ up to 12 digits using double precision. By default, our numerical evaluation is based on the use of $q_{1,2}=\left(p_{2}, p_{1}\right)$. For the checks in the case of massive $b$ quarks the result with $q_{1,2}=$ $\left(p_{2}, p_{1}\right)$ is compared with the one using any $q_{1,2}$ such as the conditions (A4) are obeyed. In the case of massless $b$ quarks it is simplest to take $q_{1,2}=\left(p_{3}, p_{4}\right)$. 
This check is an important check on many ingredients that enter the calculation: the Dirac spinors, the gluon polarization vectors, the propagators, the Lorentz indices, the loop integrals. It has been used extensively in our numerical calculation.

\section{APPENDIX B: OPTIMIZATION}

Each helicity amplitude $\mathcal{A}\left(\lambda_{1}, \lambda_{2} ; \lambda_{3} \lambda_{4}\right) \equiv \mathcal{A}(\hat{\lambda})$, a $C$ number, is calculated numerically in the FORTRAN code. The price to pay is that the number of helicity amplitudes to be calculated can be large, 16 in our case for the electroweak loop part. Some optimization is necessary. The categorization of the full set of diagrams into three gauge invariant classes as shown in Sec. IIC is a first step. We have sought to write each diagram as a compact product of blocks and structures containing different properties of the amplitude. We write the amplitude according to a color ordering pattern that defines three channels. The ordering is in a one-to-one correspondence with the three channels or diagrams shown in Fig. 1. The $T$ type is the direct channel, the $U$ type is the crossed one obtained from the $T$ type by interchanging the two gluons, and the $S$ type is the one involving the triple gluon vertex. The helicity amplitude for each diagram can thus be represented as

$$
\mathcal{A}(\hat{\lambda})^{T, U, S}=\operatorname{CME}(a, b) \times C c \times F F E \times \operatorname{SME}\left(\lambda_{i}\right),
$$

where

(i) $\operatorname{CME}(a, b)$ is the color matrix element. $a, b$ are the color indices of the two initial gluons. ${ }^{3}$ The color products can be $\left(T^{a} T^{b}\right),\left(T^{b} T^{a}\right)$, or $\left[T^{a}, T^{b}\right]$ corresponding to the $3 T, U, S$ channels, respectively

(ii) $C c$ contains all the common coefficients like the strong coupling constant $g_{s}$ or factors common to all diagrams and amplitudes such as the normalization factor entering the representation of the polarization vector of the gluon; see Eq. (A3)

(iii) $F F E$, form factor element, contains all the denominators of propagators, loop functions as well as various scalar products of external momenta $\left\{p_{1}, p_{2}, p_{3}, p_{4}\right\}$, i.e., all the scalar objects which do not depend on the helicity $\lambda_{i}$

(iv) $\operatorname{SME}(\hat{\lambda})$, standard matrix element, is a product of the scalar spinor functions $A_{\lambda_{i} \lambda_{j}}, B_{\lambda_{i} \lambda_{j}}$, and $C_{\lambda_{i} \lambda_{j}}$ defined in Appendix A.

For each channel, say $\mathcal{A}(\hat{\lambda})^{T}$, the most complicated and time-consuming part is the FFE. That is why we want to factorize it out and put it in a common block so that in order to calculate all the 16 helicity configurations of $\mathcal{A}(\hat{\lambda})^{T}$ we just need to calculate $F F E$ once. This is done

\footnotetext{
${ }^{3}$ Other color indices of the bottom quarks are omitted here for simplicity
}

at every point in phase space. This kind of factorization can be easily carried out in FORM.

$\operatorname{SME}(\hat{\lambda})$ is also complicated because the bottom quark is massive and $\gamma_{5}$ occurs in the "helicity strings." Thus we have to optimize this part as well. The way we do it for all the 3 groups is as follows. In FORM, we have to find out all the generic expressions of $\operatorname{SME}(\hat{\lambda})$. There are 12 of them at tree-level and 68 at one-loop if we choose $q_{1,2}=p_{2,1}$ for the reference vectors. For instance,

$$
\begin{aligned}
S M E_{1}= & {\left[\bar{u}\left(\lambda_{3}, p_{3}\right) v\left(\lambda_{4}, p_{4}\right)\right] \times\left[\varepsilon_{\mu}\left(\lambda_{1}, p_{1}, p_{2}\right) p_{4}^{\mu}\right] } \\
& \times\left[\varepsilon_{\nu}\left(\lambda_{2}, p_{2}, p_{1}\right) p_{4}^{\nu}\right], \\
= & B M E_{1}\left(\lambda_{3}, \lambda_{4}\right) \times B M E_{2}\left(\lambda_{1}\right) \times B M E_{3}\left(\lambda_{2}\right)
\end{aligned}
$$

can be expressed in terms of 3 basic matrix elements $(B M E)$. Each $B M E$ occurs several times when calculating all the $S M E(\hat{\lambda})$. The number of $B M E$ is 31 . Each $B M E$ is written in terms of scalar spinor functions $A_{\lambda_{i} \lambda_{j}}, B_{\lambda_{i} \lambda_{j}}$, $C_{\lambda_{i} \lambda_{j}}$. All the $S M E$ or $B M E$ can be found and abbreviated in FORM. As an alternative, we can use PERL for such an operation. The FORM output is converted directly into a FORTRAN code for numerical evaluation. Needless to say, all the abbreviations of $S M E$ or $B M E$ must be put in common blocks.

To get the final result, we have to sum over all the channels. The grouping can be rearranged in terms of an Abelian part and a non-Abelian part according to

$$
\begin{aligned}
\mathcal{A}(\hat{\lambda}) & =\mathcal{A}(\hat{\lambda})^{T}+\mathcal{A}(\hat{\lambda})^{U}+\mathcal{A}(\hat{\lambda})^{S}, \\
& \equiv\left\{T^{a}, T^{b}\right\} \mathcal{A}(\hat{\lambda})^{\mathrm{Abel}}+\left[T^{a}, T^{b}\right] \mathcal{A}(\hat{\lambda})^{\text {non-Abel }},
\end{aligned}
$$

where

$$
\begin{aligned}
\mathcal{A}(\hat{\lambda})^{\text {Abel }} & =\frac{1}{2}\left(\mathcal{A}(\hat{\lambda})^{T}+\mathcal{A}(\hat{\lambda})^{U}\right), \\
\mathcal{A}(\hat{\lambda})^{\text {non-Abel }} & =\mathcal{A}(\hat{\lambda})^{S}+\frac{1}{2}\left(\mathcal{A}(\hat{\lambda})^{T}-\mathcal{A}(\hat{\lambda})^{U}\right),
\end{aligned}
$$

corresponding to the Abelian and non-Abelian parts, respectively. The amplitude squared then contains no interference term between the Abelian and non-Abelian parts:

$$
|\mathcal{A}(\hat{\lambda})|^{2}=\frac{1}{256}\left(\frac{28}{3}\left|\mathcal{A}(\hat{\lambda})^{\mathrm{Abel}}\right|^{2}+12\left|\mathcal{A}(\hat{\lambda})^{\text {non-Abel }}\right|^{2}\right)
$$

where $\frac{1}{256}=\frac{1}{4} \times \frac{1}{8} \times \frac{1}{8}$ is the spin- and color-averaging factor. 
[1] A. Djouadi, arXiv:hep-ph/0503172.

[2] R. M. Barnett, H. E. Haber, and D. E. Soper, Nucl. Phys. B306, 697 (1988).

[3] D. A. Dicus and S. Willenbrock, Phys. Rev. D 39, 751 (1989).

[4] S. Dittmaier, M. Krämer, and M. Spira, Phys. Rev. D 70, 074010 (2004).

[5] S. Dawson, C. B. Jackson, L. Reina, and D. Wackeroth, Phys. Rev. D 69, 074027 (2004).

[6] S. Dawson, C. B. Jackson, L. Reina, and D. Wackeroth, Mod. Phys. Lett. A 21, 89 (2006).

[7] C. Buttar et al., arXiv:hep-ph/0604120.

[8] D. Dicus, T. Stelzer, Z. Sullivan, and S. Willenbrock, Phys. Rev. D 59, 094016 (1999); C. Balazs, H. J. He, and C. P. Yuan, Phys. Rev. D 60, 114001 (1999).

[9] R. V. Harlander and W. B. Kilgore, Phys. Rev. D 68, 013001 (2003).

[10] S. Dittmaier, M. Kramer, A. Muck, and T. Schluter, J. High Energy Phys. 03 (2007) 114.

[11] G. Gao, R. J. Oakes, and J. M. Yang, Phys. Rev. D 71, 095005 (2005).

[12] W. Hollik and M. Rauch, AIP Conf. Proc. 903, 117 (2007).

[13] S. Dawson and C. B. Jackson, Phys. Rev. D 77, 015019 (2008).

[14] LEP Collaborations ALEPH, DELPHI, L3, OPAL, and LEP Electroweak Working Group, arXiv:hep-ex/0612034.

[15] G. Bélanger, F. Boudjema, J. Fujimoto, T. Ishikawa,
T. Kaneko, K. Kato, and Y. Shimizu, Phys. Rep. 430, 117 (2006).

[16] J. A. M. Vermaseren, arXiv:math-ph/0010025

[17] G. 't Hooft and M. Veltman, Nucl. Phys. B153, 365 (1979); G. Passarino and M. J. G. Veltman, Nucl. Phys. B160, 151 (1979).

[18] T. Hahn and M. Perez-Victoria, Comput. Phys. Commun. 118, 153 (1999); G. J. van Oldenborgh and J. A. M. Vermaseren, Z. Phys. C 46, 425 (1990).

[19] S. Kawabata, Comput. Phys. Commun. 88, 309 (1995).

[20] A. C. Genz and A. A. Malik, J. Comput. Appl. Math. 6, 295 (1980); http://wwwasdoc.web.cern.ch/wwwasdoc/ shortwrupsdir/d120/top.html.

[21] A. Denner and S. Dittmaier, Nucl. Phys. B658, 175 (2003).

[22] T. Hahn and M. Rauch, Nucl. Phys. B, Proc. Suppl. 157, 236 (2006); arXiv:hep-ph/0601248.

[23] A. Pukhov, CALCHEP, http://www.ifh.de/ pukhov/ calchep.html.

[24] http://user.pa.msu.edu/wkt/cteq/cteq6/cteq6pdf.html.

[25] K.A. Assamagan et al. (Higgs Working Group Collaboration), arXiv:hep-ph/0406152.

[26] L. D. Landau, Nucl. Phys. 13, 181 (1959).

[27] B. A. Kniehl, C. P. Palisoc, and A. Sirlin, Nucl. Phys. B591, 296 (2000).

[28] R. Kleiss and W. J. Stirling, Nucl. Phys. B262, 235 (1985).

[29] A. Ballestrero and E. Maina, Phys. Lett. B 350, 225 (1995). 